\title{
Variaciones ultraestructurales inducidas por Cromo (VI) en hojas de jacinto acuático (Eichhornia crassipes)
}

\author{
Willy Montoya-Palomino ${ }^{1, *}$, Enrique Javier Peña-Salamanca ${ }^{2}$ y Gerardo Andrés Torres- \\ Rodríguez ${ }^{3}$ \\ ${ }^{1}$ Carrera 26. No. 32 a 22. Barrio San Benito. Cali, Valle del Cauca-Colombia. \\ 2 Departamento de Biología. Facultad de Ciencias Naturales y Exactas. Universidad del Valle. Cali, Colombia. \\ ${ }^{3}$ Unidad de Microscopia Electrónica, Universidad del Cauca. Popayán Cauca Colombia. \\ * Autor responsable de la correspondencia: willymontoya@gmail.com
}

Recibido: 25/02/2012

Aceptado: 04/12/2014

\begin{abstract}
Ultraestructural changes induced by chromium (VI) in water hyacinth (Eichhornia crassipes) leaves

In this study cellular alterations induced by $\mathrm{Cr}$ (VI) were determined on the water hyacinth, Eichhornia crassipes [Mart.] SolmLaubach, through high resolution optical microscopy and transmission electron microscopy. Mature plants of $E$. crassipes were acclimated and exposed to 0,10, 20 and $30 \mathrm{mg} / \mathrm{l}$ of chromium (VI) during 144 hours under controlled conditions in a greenhouse. Ultrastructural and tisular damages induced by chromium were determined comparing control (no metal) and $\mathrm{Cr}$ exposed plants. E. crassipes showed damages on the structure and distribution of thylakoid membranes by exposition higher to $20 \mathrm{mg} / \mathrm{l}$. It was observed that plants exposed to $20 \mathrm{mg} / \mathrm{l}$ of $\mathrm{Cr}$ (VI) showed chloroplast membrane disruption and low stacking grana. It was not observed ultrastructure damages in E. crassipes chloroplasts in chromium concentrations below to $20 \mathrm{mg} / \mathrm{l}$. It was observed a high $\mathrm{Cr}(\mathrm{VI})$ accumulation on water hyacinth root tissues exposed to 20 and $30 \mathrm{mg} / \mathrm{l}$.
\end{abstract}

Key words: Ultrastructure, Eichhornia crassipes, chromium, transmission electron microscopy, bioaccumulation.

\section{RESUMEN}

Variaciones ultraestructurales inducidas por Cromo (VI) en hojas de jacinto acuático (Eichhornia crassipes)

En el presente estudio se determinaron las alteraciones ultraestructurales en Eichhornia crassipes [Mart.] Solm-Laubach inducidas por cromo, a través de microscopía óptica de alta resolución y del microscopio electrónico de transmisión. Las plantas maduras de jacinto acuático fueron aclimatadas y sometidas a 0,10, 20 y $30 \mathrm{mg} / \mathrm{l}$ de cromo (VI) durante 144 horas de exposición al metal, bajo condiciones controladas en invernadero Las alteraciones ultraestructurales y tisulares inducidas por cromo se determinaron a través de observaciones comparativas entre los organismos control y las plantas tratadas. E. crassipes mostró daños en la disposición y estructura de las membranas tilacoidales a partir de exposiciones de $20 \mathrm{mg} / \mathrm{l}$. Se observó que las plantas expuestas a $20 \mathrm{mg} / \mathrm{l}$ de Cr (VI) presentaban una ruptura de la membrana del cloroplasto y grana con pocos apilamientos. No se observaron daños ultraestructurales a concentraciones de cromo menores de $20 \mathrm{mg} / \mathrm{l}$. Se observó una alta bioacumulación de Cr (VI) en tejidos radiculares de jacinto acuático expuestos a 20 y $30 \mathrm{mg} / \mathrm{l}$.

Palabras clave: Ultraestructura, Eichhornia crassipes, cromo, microscopia electrónica de transmisión, bioacumulación.

\section{INTRODUCCIÓN}

El cromo es un metal de transición que se vierte en cantidades apreciables a los sistemas hídri- cos, por las industrias galvánica y curtiembres, para dar durabilidad al acero y otros materiales y evitar pudrición en la madera y el cuero (Pettine et al., 2006; Maine et al., 2004; Barnhart, 
1997). La forma trivalente del cromo tiene actividad biológica a bajas concentraciones, es poco soluble, muy estable y su capacidad para transportar electrones es baja (Pettine et al., 1998). El cromo hexavalente no tiene actividad biológica conocida, es 100 veces más tóxico que el Cr (III) y se transporta al interior celular como análogo del ión sulfato; genera cáncer, mutaciones y puede causar la muerte. El $\mathrm{Cr}$ (III) es oxidado en ambientes naturales a $\mathrm{Cr}(\mathrm{VI})$, la forma más tóxica del metal con implicaciones desfavorables para la biota asociada (Ksheminska et al., 2005).

Eichhornia crassipes (Monocotiledónea, Pontederiaceae), el jacinto acuático, es una macrófita flotante dulceacuícola, distribuida por todo el cinturón tropical y subtropical del globo. Se dispersó desde el Amazonas brasileño (Carignan et al., 1994) y es ahora una plaga (Xie \& Yu, 2003). Crece rápidamente, forma masas compactas y genera problemas por taponamiento de sistemas fluviales.

Estudios previos sobre la estructura celular de E. crassipes han demostrado una disminución en el apilamiento de membranas tilacoidales, incremento del volumen estromático y presencia de plastoglóbulos, en plantas recogidas de aguas naturales contaminadas con materia orgánica, metales pesados y otros tipos de contaminantes industriales (Lage-Pinto et al., 2008). Kumar-Panda (2007), observó acortamiento de la raíz por aumento en el crecimiento de las células corticales y daño en la membrana de las células radiculares por generación de peróxido de hidrógeno $\left(\mathrm{H}_{2} \mathrm{O}_{2}\right)$ y radicales superóxido $\left(\mathrm{O}_{2}^{-}\right)$, que deviene en la oxidación de los lípidos membranales por radicales hidróxilo $\left(\mathrm{OH}^{-}\right)$y finalmente el ingreso del $\mathrm{Cr}(\mathrm{VI})$ a la planta. La exposición de plantas a concentraciones de $\mathrm{Cr}$ (VI) desde 1 a $250 \mathrm{mg} / \mathrm{l}$, induce clorosis en rábano (Dube et al., 2005), disminuye la concentración de clorofila en junco (Gruber et al., 2008) y genera pérdida de la integridad general de la hoja y necrosis en jacinto acuático (Brito-Paiva et al., 2009). La concentración normal de cromo en plantas está entre 20 y $200 \mu \mathrm{g} / \mathrm{kg}$ de peso seco (PS), pero plantas que crecen en suelos serpentinos como Leptospermum scoparum, Sutera fodina y Dicoma niccolifera pueden acumular en tejidos foliares has- ta $20 \mathrm{~g} / \mathrm{kg}$ (PS), aproximadamente un $33 \%$ de la concentración edáfica del metal. Con relación a la acumulación incremental de cromo en las redes tróficas, resultados publicados sugieren que su alta toxicidad a bajas concentraciones impide la biomagnificación, ya que los organismos mueren antes de ser asimilados por el siguiente nivel trófico, con lo que el cromo pasaría de nuevo al sedimento, que se comporta como sumidero (Zayed \& Terry, 2003).

Estudios recientes sobre el Cr (VI) establecieron que en la Laguna de Sonso, Valle del CaucaColombia, los niveles de cromo en sedimentos son de $70 \mathrm{mg} / \mathrm{kg}$ y de $88 \mu \mathrm{g} / \mathrm{l}$ en agua, valores de sistemas naturales considerados como mediana y fuertemente contaminados, respectivamente (Peña et al., 2012; Varón-López, 2009; Anderson \& Azcárate, 2005; Gischler, 2005). Las plantas de jacinto acuático o buchón de agua (denominación local), presentes en la laguna de Sonso, adsorben aproximadamente el $10 \%$ de este cromo. Las iniciativas tendientes al mejoramiento de la capacidad de tolerancia/bioacumulación de cromo por E. crassipes, así como la determinación de daños en la estructura íntima de los tejidos de esta planta son importantes en la medida en que disminuyen la biodisponibilidad del cromo a otros niveles tróficos y favorecen su reducción a la forma menos tóxica $\mathrm{Cr}$ (III) (Ksheminska et al., 2005).

Diversos trabajos han evaluado la capacidad de Eichhornia crassipes para tolerar altas concentraciones de metales, y se ha reconocido su capacidad para ser usado en procesos de fitoextracción-volatilización y bioindicación (Gardea-Torresdey et al., 2010; Ebel et al., 2007). En estos estudios se ha determinado la respuesta enzimática (catalasa), la actividad fotosintética medida a través de la fluorescencia de la clorofila y sus variaciones ultraestructurales (Mangabeira et al., 2004), midiendo daños a nivel citosólico y vascular, al detectar cambios en tejidos foliares o depósitos electrodensos en tejidos radiculares. $E$. crassipes puede reducir la forma tóxica $\mathrm{Cr}$ (VI) en $\mathrm{Cr}$ (III) y acumularla (Mandiwana et al., 2007).

El jacinto acuático ha sido estudiado para determinar su capacidad de acumular y transformar sustancias tóxicas del agua, dada su gran resis- 
tencia a altas concentraciones y distintas especies químicas. El cromo, proveniente principalmente de las industrias peleteras, es vertido en dos especies químicas principales, las formas trivalente (Cr (III)) y hexavalente (Cr (VI)) (Zayed \& Terry, 2003). Cr (VI) es muy tóxico y existe poca información sobre el daño que ocasiona a nivel ul- traestructural en las plantas con potencial biorremediador, como el jacinto acuático. La presencia de daños a nivel ultraestructural en plantas tratadas con Cr (VI) sería un importante indicador precoz de los límites de tolerancia de la planta a la sustancia en condiciones de campo como posible agente fitoacumulador de $\mathrm{Cr}(\mathrm{VI})$. La for-
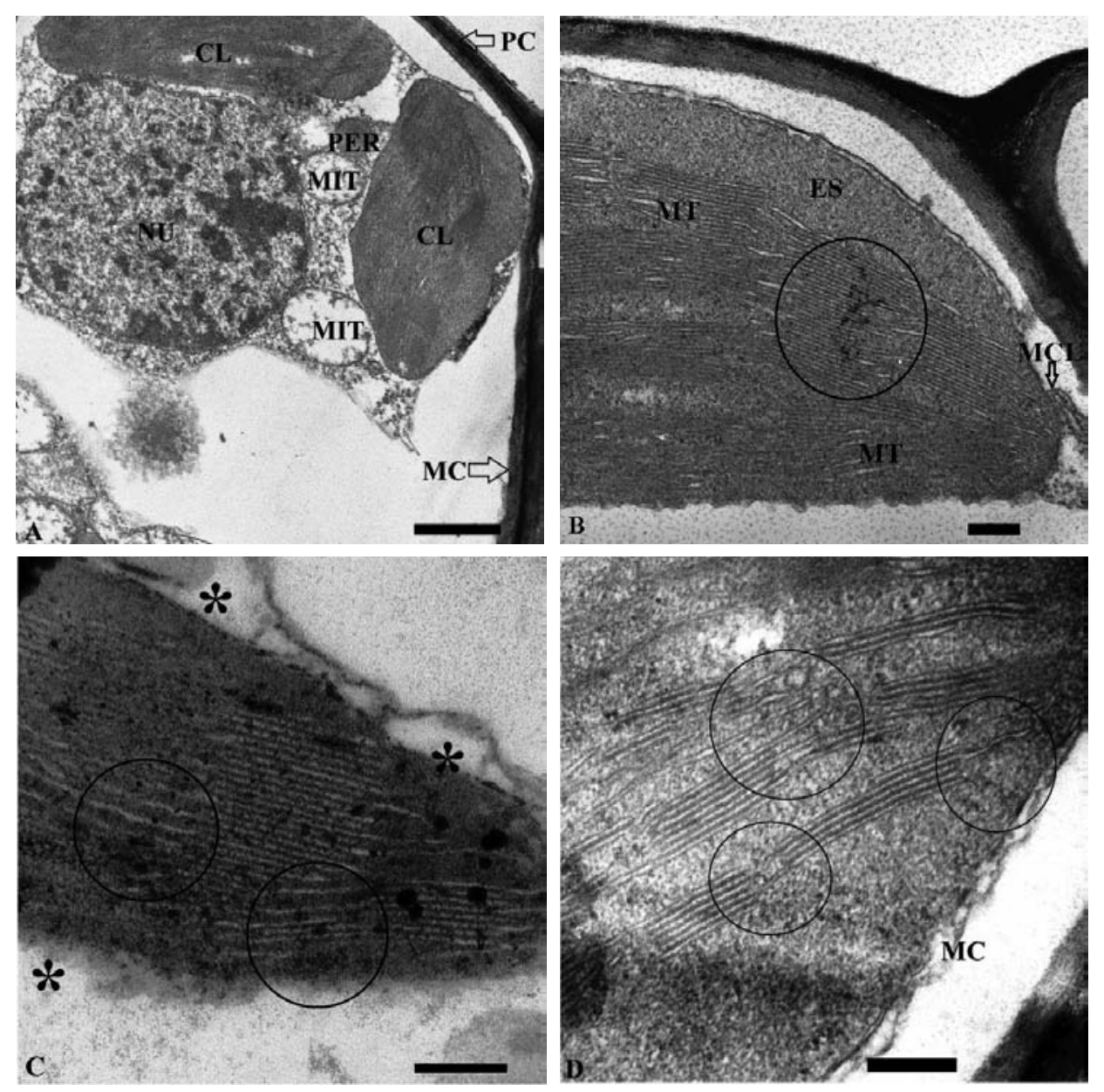

Figura 1. A. Micrografía TEM de célula de jacinto acuático sin tratar, donde se muestran cloroplastos (CL), mitocondrias (MIT), el núcleo (NU) y un peroxisoma (PER). PC y MC denotan la pared y membrana celular respectivamente. Barra escalar de $0.5 \mu$ m. B. Micrografía TEM de cloroplasto de jacinto acuático sin tratar. Se muestran las membranas tilacoidales (MT), la membrana del cloroplasto (MCL) y su matriz interior o estroma (ES). Barra escalar de $200 \mathrm{~nm}$. C. Micrografía TEM de cloroplasto de jacinto acuático tratado con $20 \mathrm{mg} / \mathrm{l}$ de Cr (VI). Los círculos muestran membranas tilacodides desapiladas, y los asteriscos membranas del cloroplasto discontinuas. Barra escalar de $200 \mathrm{~nm}$. D. Micrografia TEM de cloroplasto de jacinto acuático tratado con $30 \mathrm{mg} / \mathrm{l}$ de $\mathrm{Cr}$ (VI). Los círculos muestran membranas tilaodides desapiladas, y los asteriscos membranas del cloroplasto discontinuas. Barra escalar de $200 \mathrm{~nm}$. A. TEM micrograph of control water hyacinth cell. Micrograph shows chloroplasts (CL), mitochondrias (MIT), nucleus (NU) and peroxisome (PER), Scale bar $0.5 \mu \mathrm{m}$. B. TEM micrograph of young chloroplast from control water hyacinth plant. Micrograph shows tylacoid membranes (MC), chloroplast membranes (MCL), and inside matrix or estroma (ES). Scale bar $200 \mathrm{~nm}$. C. TEM micrograph of chloroplast from plants exposed to $20 \mathrm{mg} / \mathrm{l} \mathrm{of} \mathrm{Cr}(\mathrm{VI})$. Circles show unstacked tylacoid membranes, and asterisks disrupted chloroplast membranes (MCL). Scale bar $200 \mathrm{~nm}$. D. TEM micrograph of chloroplast from plants exposed to $30 \mathrm{mg} / \mathrm{l}$ of $\mathrm{Cr}$ (VI). Circles show unstacked tylacoid membranes, and asterisks disrupted chloroplast membranes (MCL). Scale bar 200 nm. 
ma hexavalente del cromo (Cr (VI)) es la especie química que con mayor frecuencia se elimina sin tratar en los procesos de curtición (curtiembre) del cuero, electroplateado y en efluentes domésticos. Cr (VI) es a su vez poco estudiada en términos de su capacidad para alterar la ultraestructura celular del jacinto acuático, macrófita de amplio uso como agente biorremediador de sustancias tóxicas en el medio acuático (Lage-Pinto et al. 2008; Malik, 2007). Cr (VI), penetra fácilmente las membranas celulares y es reducida a la forma de cromo trivalente. Su capacidad de daño a nivel celular está relacionada con el alto potencial redox de los iones cromato en las membranas (Giri \& Patel, 2011; Sankar-Ganesh et al., 2008).

Se ha demostrado ampliamente la capacidad de tolerancia de sustancias tóxicas del agua por parte del jacinto acuático, dada su resistencia a altas concentraciones y distintas especies químicas (Azizur-Rahman \& Hasegawa 2011; Agunbiade et al., 2009). También se han evaluado los daños ultraestructurales inducidos en cloroplastos con relación a la cercanía de fuentes antropogénicas de cromo, como industrias y ciudades. Estos estudios usaron plantas coleccionadas en campo, donde la determinación exacta de la especie química del metal y la concentración de cromo que generó estos daños es compleja (Vitória et al. 2011; Lage-Pinto et al. 2008; Sanita di Toppi et al. 2007; Mangabeira et al. 2004).

En el presente estudio se evalúan los cambios ultraestructurales y la capacidad de acumulación de $\mathrm{Cr}$ (VI) en E. crassipes a partir de un bioensayo con Cr (VI). Las respuestas de la planta son de utilidad para establecer líneas base de límites de vertimientos de cromo a fuentes de agua natural y su correlación con daños en la biología de la planta.

\section{MATERIALES Y MÉTODOS}

\section{Selección del material vegetal, limpieza y aclimatación}

Se dispusieron doce (12) plantas de E. crassipes procedentes de un estanque surtido con agua potable en acuarios de 401 con solución Hoagland modificada (Hoagland \& Arnon, 1950; Wang \& Jia, 2009), dentro del invernadero de la Estación Experimental del Departamento de Biología en el campus central de la Universidad del Valle. Durante un período de dos (2) semanas de aclimatación, el medio de cultivo fue cambiado cada tres (3) días. Pasado este tiempo, se iniciaron los bioensayos con $\mathrm{Cr}$ (VI). Las plantas fueron expuestas durante 144 horas a cuatro tratamientos: $0 \mathrm{mg} / \mathrm{l}$ de $\mathrm{Cr}$ (VI), como el tratamiento control y a concentraciones nominales de 10, 20 y $30 \mathrm{mg} / 1$ de Cr (VI). Para cada tratamiento se dispusieron 3 réplicas en recipientes distintos. Las soluciones de $\mathrm{Cr}$ (VI) fueron preparadas a partir de bicromato de potasio (MERCK, Alemania) disuelto en agua desionizada.

\section{Análisis de la ultraestructura}

Tres biopsias de tejido foliar por tratamiento fueron procesadas para microscopía electrónica de transmisión (MET), de acuerdo con los protocolos de Dykstra \& Reuss (2003): fijación con glutaraldehído al $2 \%$ y post-fijación con tetróxido de osmio al $2 \%$; deshidratación en escala ascendente de alcoholes; imbibición previa de las muestras deshidratadas con acetona e imbibición con resina; endurecimiento de las muestras durante 48 horas a $72{ }^{\circ} \mathrm{C}$; pulido de bloques, cortes semifinos y observación en microscopía óptica del alta resolución (MOAR, coloración con azul de toluidina); obtención de cortes ultrafinos, tinción de contraste (acetato de uranilo y citrato de plomo), observación, captura gráfica en MET y determinación de las diferencias entre las membranas tilacoidales en cloroplastos de plantas tratadas, respecto de las membranas tilacoidales de cloroplastos en plantas control.

\section{Análisis de bioacumulación por Espectroscopía de Absorción Atómica (EAA)}

Las biopsias fueron procesadas en el laboratorio de análisis industriales de la Universidad del Valle en Cali. La determinación analítica de la concentración de metal acumulado, se llevaron a cabo mediante la técnica de Espectrometría de Absorción Atómica por horno de grafito (espec- 
trofotómetro Shimatzu AA. 660), previa digestión ácida de veinte (20) gramos (PS) de cada tipo de tejido estudiado. La tasa de acumulación del metal en la planta fue calculada a partir del modelo de absorción de Langmuir (V), mediante la ecuación $V=\left(C_{i}-C_{f}\right) / N \times T$, donde $V=$ tasa de acumulación, $C_{i}$ y $C_{f}$ las concentraciones de metal a tiempo cero y a 144 horas, $N$, la cantidad de buchón en peso seco y $T$ el tiempo de exposición (Wang \& Lewis, 1997). Los valores de acumulación obtenidos se expresaron en miligramos de $\mathrm{Cr}$ (VI) acumulado por cada kilogramo de tejido expuesto al metal.

\section{Análisis de datos}

Se realizó un análisis de varianza (ANOVA) de dos vías teniendo como factores: la concentración de $\mathrm{Cr}$ (VI) con cuatro niveles $0,10,20 \mathrm{y}$ $30 \mathrm{mg} / \mathrm{l}$ y el tipo de tejido (hojas y raíces), con un total de 12 plantas $(N=12)$. Posteriormente, se realizó una prueba Post-Anova de Tukey para evaluar diferencias entre las concentraciones (tratamientos) y el tipo de tejido. Los análisis estadísticos se probaron a un nivel de significancia del $95 \%$ con el programa Statistica versión 7 (2004). Las microfotografías de Eichhornia crassipes contaminado con cromo se analizaron respecto de plantas control, para la determinación de daños en la ultraestructura celular.

\section{RESULTADOS}

\section{Daños inducidos por cromo (VI) en la ultraestructura del cloroplasto de Eichhornia crassipes}

La figura 1A muestra la disposición y forma de los organelos subcelulares de plantas de Eichhornia crassipes sin tratar. Se observan mitocondrias (MIT), cloroplastos (CL), el núcleo (NU) y un peroxisoma (PER). Se observan también la membrana y pared celular (MC y PC, respectivamente).

La figura $1 \mathrm{~B}$ muestra un cloroplasto de plantas control de E. crassipes, con sus grandes apilamientos tilacoidales y membrana del cloroplasto bien delimitada.
Tabla 1. Presencia/ausencia de daños ultraestructurales inducidos por $\mathrm{Cr}$ (VI) en subestructuras del cloroplasto de Eichhornia crassipes $(N=12$, tres observaciones por tratamiento) $(p<0.05)$. Presence or absence of ultraestructural damages induced by $\mathrm{Cr}$ (VI) on chloroplasts substructures of Eichhornia crassipes $(\mathrm{N}=12$, three observations per treatment $)(\mathrm{p}<0.05)$.

\begin{tabular}{lccc}
\hline Tejido & Control* & $20 \mathrm{mg} / \mathrm{l}$ & $30 \mathrm{mg} / \mathrm{l}$ \\
\hline MC** & 1 & 7 & 8 \\
MT*** & 0 & 6 & 4 \\
Grana & 0 & 2 & 6 \\
Total & 1 & 15 & 18 \\
\hline * Los números en la tabla corresponden con los daños encontrados por tipo \\
de tejido analizado. \\
** MC: membrana del cloroplasto. \\
*** MT: membrana tilacoidal.
\end{tabular}

En la figura 1C se muestra un cloroplasto de E. crassipes, tratado con $20 \mathrm{mg} / \mathrm{l}$ de $\mathrm{Cr}$ (VI). Se observan (círculos) las membranas tilacoidales separadas y depósitos electrodensos en la porción derecha de la micrografía; los asteriscos resaltan las zonas de la membrana de cloroplastos con interrupciones. Es evidente la presencia de glóbulos osmiofílicos (puntos obscuros en la porción derecha de la micrografía) en el estroma de los cloroplastos y la membrana externa del cloroplasto rota.

En la figura 1D se muestra un cloroplasto de E. crassipes tratado con $30 \mathrm{mg} / \mathrm{l}$ de Cr (VI). Los círculos denotan zonas de poco apilamiento de las membranas tilacoidales y la separación de los grana. Se observa igualmente (MC) la membrana del cloroplasto con interrupciones y zonas de grosor irregular. Los daños observados en los tratamientos analizados se muestran en la Tabla 1. Los valores dentro de la tabla corresponden a la observación de la presencia de daño en la estructura mencionada para los tratamientos control, $20 \mathrm{mg} / \mathrm{l}$ y $30 \mathrm{mg} / \mathrm{l}$ de $\mathrm{Cr}$ (VI), como resultado de las observaciones de las biopsias de plantas por cada tratamiento durante el tiempo de exposición de 144 horas.

\section{Acumulación de cromo (VI) por $E$. crassipes en condiciones de bioensayo}

Se encontraron diferencias significativas $(p<0.05)$ de la concentración de Cr (VI) entre hojas y raí- 


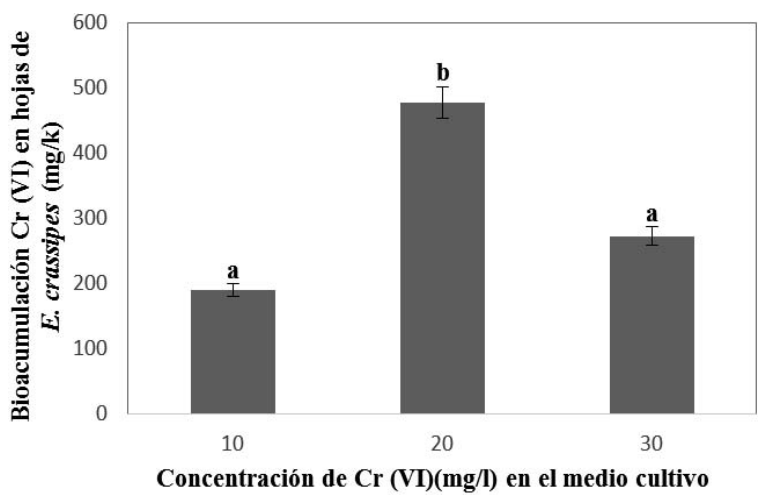

Figura 2. Valores promedio ( \pm desviación estándar) de la concentración de cromo en tejidos de hoja de Eichhornia crassipes expuestas a 10, 20 y $30 \mathrm{mg} / \mathrm{l}$. Las letras sobre las barras indican las diferencias entre tratamientos $(p<0.05)$. Mean values ( \pm standard deviation) of chromium concentrations in leaf tissues of Eichhornia crassipes exposed to 10, 20 and $30 \mathrm{mg} / \mathrm{l}$. Letters above bars show differences between treatments $(\mathrm{p}<0.05)$.

ces de jacinto acuático expuestas al metal. Los datos de la prueba de análisis de varianza (ANOVA) y Post-Anova de Tukey (Tabla 2) mostraron que para los tejidos foliares existen diferencias de la acumulación del metal entre las concentraciones de 10 y 20 mg/l respectivamente (Fig. 2).

Para el caso de la acumulación del metal en los tejidos radiculares sólo se detectaron diferencias significativas entre las concentraciones de 10 y $20 \mathrm{mg} / \mathrm{l}$ respectivamente (Fig. 3). La acumulación de $\mathrm{Cr}$ (VI) en las plantas presentó concentraciones superiores entre uno y dos órdenes de magnitud a las que fueron expuestas (Fig. 2 y 3 ).

\section{DISCUSIÓN}

\section{Daños en la ultraestructura del cloroplasto}

Los resultados obtenidos en este estudio muestran que el análisis de la ultraestructura del cloroplasto es una herramienta útil en la determinación de daños tempranos por metales en el aparato fotosintético y que su identificación puede ayudar en la prevención de efectos mayores en la planta.

Se observa que en el tratamiento donde se somete a la planta a $20 \mathrm{mg} / \mathrm{l}$ de $\mathrm{Cr}$ (VI) existen variaciones importantes respecto de la conformación de las membranas tilacoidales (poca intensi-
Tabla 2. Resultados del análisis Anova para dos factores: concentración y tipo de tejido (hoja y raíz), para probar diferencias entre los valores de acumulación de $\mathrm{Cr}$ (VI) en Eichhornia crassipes. Results of the two-way Anova test: metal concentration and plant tissue (leaf and root) for Cr (VI)'s accumulation in Eichhornia crassipes.

\begin{tabular}{cccc}
\hline Fuente & GL & F & P \\
\hline Factor 1* & 3 & 21.63 & 0.0002 \\
Factor 2** & 1 & 124.75 & 0.0001 \\
Interacción & 3 & 18.87 & 0.0002 \\
Error & 16 & & 0.0003 \\
Total & 23 & & 0.0001 \\
\hline
\end{tabular}

$S=11.5 ; R^{2}=94.9 \% ; R^{2}$ (ajustado) $=92.2 \%$

* Concentración de $\mathrm{Cr}(\mathrm{VI})$.

** Tipo de tejido.

dad) y sus grana. Además, la membrana del cloroplasto aparece simple, difusa y rota y la presencia de glóbulos osmiofílicos es notable. Estas estructuras son depósitos de membranas tilacoidales en proceso de resorción y/o exporte del cloroplasto (Brito-Paiva et al., 2009). Al aumentar la concentración de cromo a $30 \mathrm{mg} / \mathrm{l}$ de $\mathrm{Cr}$ (VI) se mantienen los daños presentes en el tratamiento intermedio, pero los grana tienen un apilamiento menor. Otras organitos como mitocondrias, vacuolas y núcleo mantienen sus estructuras características en el rango de concentraciones estudiado. Macrófitas como el junco (Juncus effusus) y la espinaca acuática (Ipomonea aquatica) muestran, en rangos similares al estudiado, daños a nivel fotosintético, en términos de la concentración

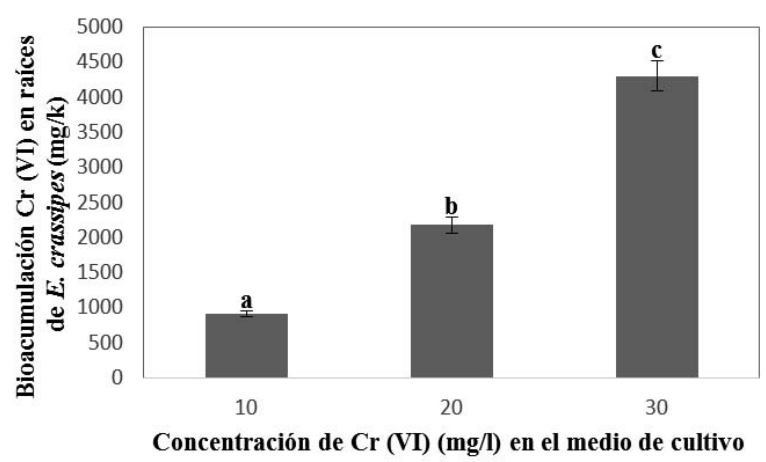

Figura 3. Valores promedio ( $n=3 ; \pm$ desviación estándar) de la concentración de cromo en tejidos de raíz de Eichhornia crassipes expuestas a 10, 20 y $30 \mathrm{mg} / \mathrm{l}$. Las letras sobre las barras indican las diferencias entre tratamientos $(p<0.05)$. Mean values ( \pm standard deviation) of chromium concentrations in root tissue of Eichhornia crassipes exposed to 10, 20 y $30 \mathrm{mg} / \mathrm{l}$. Letters above bars show differences between treatments $(\mathrm{p}<0.05)$. 
de clorofilas $a, b$ y carotenoides, con manifestaciones externas de daño, v. g. clorosis (Chen et al., 2010; Gruber et al., 2008) no evidentes en las hojas de jacinto acuático analizadas. Se ha corroborado igualmente que un número mayor de membranas tilacoidales individuales, como el caso de los daños observados, genera desbalances en el proceso de captación y transporte de electrones entre los fotosistemas (Lage-Pinto et al., 2008). Además, modificaciones en la forma y el número de las células del parénquima esponjoso y en palizada han sido observadas en hojas de plantas de mostaza india (Brassica juncea) y fríjol expuestas a Cr (VI) (Han et al., 2004).

\section{Comportamiento de la Bioacumulación en órganos de $E$. crassipes}

Eichhornia crassipes acumuló en promedio trece veces más cromo en raíces que en las partes aéreas, como se ha demostrado extensivamente en estudios sobre el jacinto acuático y otras macrófitas (Benítez et al., 2011; Peña-Salamanca et al., 2011; Brito-Paiva et al., 2009; Vardanyan \& Ingole, 2006; Paris et al., 2005). Al parecer, las plantas acuáticas reducen el Cr (VI) a Cr (III), antes de traslocarlo a las partes aéreas, en caso de exposición crónica a altas concentraciones (Weerasinghe et al., 2008). Sólo alrededor de un $10 \%$ del cromo biodisponible es translocable a la parte aérea de las plantas (Zayed \& Terry, 2003). El comportamiento bioacumulador de E. crassipes hace que se use de forma extensiva para extraer sustancias tóxicas de los cuerpos de agua donde vive (Giri et al., 2012, Patel, 2012, Vitória et al., 2011, Agunbiade et al., 2009). Uno de los factores que influye en la alta acumulación de cromo en raíces de E. crassipes consiste en la presencia de grupos cargados negativamente (carboxilo, sulfonato, fosforilo, amida e imidazol), presentes en las moléculas de celulosa y proteínas de transporte de la pared celular. Tanto moléculas de la pared celular como biopelículas de microorganismos intervienen en la fase rápida del proceso de acumulación de cromo por E. crassipes, la llamada bioadsorción, sin gasto de energía intracelular y muy eficiente a bajas concentraciones (SankarGanesh et al. 2008, Malik, 2007, Zayed \& Terry,
2003.). A exposiciones crónicas y/o altas concentraciones sobreviene la bioacumulación propiamente dicha, en la que los acarreadores de iones hierro de la membrana celular canalizan cromo al interior celular y, posteriormente, su ascenso hasta las hojas (Chojnacka, 2010).

Existe una variación notable en la concentración tisular de $\mathrm{Cr}$ (VI) de E. crassipes sometido al tratamiento medio (20 mg/l de $\mathrm{Cr}$ (VI), respecto de la bioacumulación a otras concentraciones y tiempos de exposición observados en la bibliografía. El valor de bioacumulación disminuye en las partes aéreas con capacidad fotosintética de la planta hasta los $20 \mathrm{mg} / \mathrm{l}$, contrariamente a lo ocurrido con las raíces, donde la bioacumulación aumenta en el rango de concentraciones analizado. Varios estudios demuestran la capacidad del jacinto acuático para retener y transformar en sus raíces sustancias tóxicas que podrían dañar los tejidos fotosintéticos, vitales para la planta; esta respuesta se genera sin alterar drásticamente su capacidad de intercambio iónico con el medio (Brito-Paiva et al., 2009; Espinoza-Quiñones et al., 2009).

La respuesta de la planta a una exposición y concentración moderadamente alta es típica de la fase de recuperación descrita por Larcher (1995) para plantas en condiciones de perturbación. Los resultados del estudio resaltan la capacidad de $E$. crassipes como un organismo hiperacumulador de cromo, sustentado también por otros trabajos donde se demuestra que acumula el $0.1 \%$ de $\mathrm{Cr}$ (PS) (Espinoza-Quiñones et al., 2009). El rango de acumulación de cromo en plantas es muy amplio y va desde $40 \mu \mathrm{g} / \mathrm{g}$ (PS) para el haba (Vicia faba) y la col (Brassica napus) (Zayed \& Terry, 2003) hasta 14.7 y 13.3 g/g (PS) en la microalga verde filamentosa Spirogyra sp y la macrófita Ipomonea aquatica, respectivamente (Chen et al., 2010; Ospina-Alvarez et al., 2006; Gupta et al., 2001).

Se evidenció una correlación entre el aumento de la concentración de Cr (VI) en raíces con la atenuación del daño en la ultraestructura del cloroplasto. Estos resultados sugieren que Eichhornia crassipes usaría una estrategia de compartimentalización radicular (pared externa, vacuolas de células corticales), evitando el ingreso a la par- 
te aérea de la planta del Cr (VI) y la generación de radicales libres que dañan los cloroplastos. Cuando la capacidad de acumulación radicular de cromo está comprometida, es decir, el número de sitios de unión de metal es menor que el número de cationes en el medio, el cromo fluye vía xilema hasta las hojas causando perturbación oxidativa en membranas, disminuyendo el crecimiento y acumulándose (Zayed \& Terry, 2003).

\section{CONCLUSIONES}

De acuerdo con los resultados encontrados en este trabajo se concluye que los daños inducidos por cromo en la ultraestructura del cloroplasto de Eichhornia crassipes se dan principalmente en la difusión y discontinuidad de las membranas del cloroplasto y membranas tilacoidales con poca o ninguna formación de grana. A concentraciones a partir de $20 \mathrm{mg} / \mathrm{l}$, la planta experimentó una acumulación del metal en los tejidos aéreos y consecuentes efectos sobre la ultraestructura del cloroplasto. Los patrones de acumulación de $\mathrm{Cr}$ (VI) en E. crassipes demuestran que la planta presenta mayor capacidad de acumulación de metal en los tejidos radiculares. Se propone que E. crassipes tolera concentraciones inferiores o iguales a $10 \mathrm{mg} / \mathrm{l}$ sin alterar considerablemente la estructura del cloroplasto, considerándose como un indicador temprano de daño inducido por cromo (VI).

\section{AGRADECIMIENTOS}

Este estudio se desarrolló en el marco del proyecto "Daños en la Ultraestructura Celular Inducidos por Metales Pesados en la Biota Acuática y Poblaciones Humanas Asociadas a la Laguna de Sonso, Valle del Cauca-Colombia, Código 1106335-18552, Contrato RC NO 202-2006", cofinanciado por COLCIENCIAS y ejecutado por la Universidad del Valle, la Universidad del Cauca y la Universidad Autónoma de Occidente. Reconocimientos especiales a los biólogos Patricia Mosquera de la Unidad de Microscopía Electrónica de la Universidad del Cauca y Efrén Muñoz del Laboratorio de Microscopía Electrónica de la
Universidad Nacional de Colombia, Sede Palmira, por su enseñanza de las técnicas de obtención de cortes ultrafinos, tinción, captura y revelado de micrografías electrónicas de transmisión, y al biólogo Diego Hernández por su apoyo en el desarrollo de las pruebas y análisis estadísticos.

\section{BIBLIOGRAFÍA}

AGUNBIADE, F. O., BAMIDELE, I. OLU-OWOLABI, B. KAYODE \& O. ADEBOWALE. 2009. Phytoremediation Potential of Eichhornia crassipes in Metal-Contaminated Coastal Water. Bioresource Technology, 100: 4521-4526

ANDERSON, K. \& S. E. AZCÁRATE. 2005. Strategic Environmental Assessment Pre-Study of SonsoLagoon, (Colombia). TRITA-LWR Master Thesis. Sveriges Största Tekniska Universitet. Estocolmo.

AZIZUR-RAHMAN, M. \& H. HASEGAWA. 2011. Aquatic Arsenic: Phytoremediation Using Floating Macrophytes. Chemosphere, 83: 633-646

BARNHART, J. 1997. Chromium Chemistry and Implications for Environmental Fate and Toxicity. Soil and Sediment Contamination: An International Journal, 6(6): 561-568.

BENÍTEZ, R., CALERO, V. E. PEÑA-SALAMANCA \& J. MARTIN, 2011. Evaluación de la cinética de acumulación de cromo en el buchón de agua (Echhornia crassipes). Biotecnología en el sector agropecuario y agroindustrial, 9: 66-73.

BRITO-PAIVA, L., J. GONCALVES DE OLIVEIRA, R. A. AZEVEDO, D. RODRIGUES-RIBEIRO, M. GOMES DA SILVA \& A. P. VITÓRIA. 2009. Ecophysiological Responses of Water Hyacinth Exposed to $\mathrm{Cr}^{+3}$ and $\mathrm{Cr}^{+6}$. Environmental and Experimental Botany, 65: 403-409.

CARIGNAN, R., J. J. NEIFF \& D. PLANAS. 1994. Limitation of Water Hyacinth by Nitrogen in Subtropical Lakes of the Paraná Floodplain (Argentina). Limnology and Oceanography, 39(2): 439443.

CHEN, J. C., K. S. WANG, H. CHEN, C. Y. LU, L. CH. HUANG, H. CH. LI, T. H. PENG \& S. H. CHANG. 2010. Phytoremediation of Cr (III) by Ipomonea aquatica (water spinach) from Water in the Presence of EDTA and Chloride: Effects of $\mathrm{Cr}$ Speciation. Bioresource Technology, 101: 30333039. 
CHOJNACKA, K. 2010. Biosorption and Bioaccumulation-The Prospects for Practical Applications. Environment International, 36: 299-307.

DUBE, B. K., P. SINHA, R. GOPAL \& C. CHATTERJEE. 2005. Chromium Phytotoxicity Alters Metabolism in Radish. Journal of Vegetable Crop Production, 10(2): 61-71.

DYKSTRA, M. J. \& L. E. REUSS. 2003. Biological Electron Microscopy. Theory, Technique and Troubleshootings. $2^{\text {th }}$ edition. Kluwer Academic/Plenum Publishers. Nueva York.

EBEL, M., M. W. H. EVANGELOU \& A. SCHAEFFER. 2007. Cyanide Phytoremediation by Water Hyacinths (Eichhornia crassipes). Chemosphere, 66: 816-823.

ESPINOZA-QUIÑNONES, F. R., N. MARTIN, G. STUTZ, G. TIRAO, S. M. PALACIO, M. A. RIZZUTTO, A. N. MODENES, F. G. SILVA Jr., N. SZYMANSKI \& A. D. KROUMOVE. 2009. Root Uptake and Reduction of Hexavalent Chromium by Aquatic Macrophytes Assessed by High-Resolution X-ray Emission. Water Research, 43: 4159-4166.

GARDEA-TORRESDEY, J. L., G. DE LA ROSA, J. R. PERALTA-VIDEA, M. MONTES, G. CRUZJIMENEZ \& I. CANO-AGUILERA. 2010. Differential Uptake and Transport of Trivalent and Hexavalent Chromium by Tumbleweed (Salsola kali). Archives of Environmental Contamination and Toxicology, 48: 225-232.

GISCHLER, CH. 2005. Pathways of Heavy Metals and Implications for Stakeholders, Sonso Lagoon, Colombia. TRITA-LWR Master Thesis. Sveriges Största Tekniska Universitet. Estocolmo.

GIRI, A. K. \& R. K. PATEL. 2011. Toxicity and Bioaccumulation Potential of $\mathrm{Cr}$ (VI) and $\mathrm{Hg}$ (II) on Differential Concentration by Eichhornia crassipes in Hydroponic Culture. Water Science \& Technology, 63(5): 899-906.

GIRI, A. K., P. RAJKISHORE \& S. MANDAL. 2012. Removal of Cr (VI) from Aqueous Solution by Eichhornia crassipes Root Biomass-Derived Activated Carbon. Chemical Engineering Journal, 185-186: 71-81.

GRUBER, H., A. WIESSNER, P. KUSCHK, M. KAESTNER \& K. J. APPENROTH. 2008. Physiological Responses of Juncus effusus (Rush) to Chromium and Relevance for Wastewater Treatment in Constructed Wetlands. International Journal of Phytoremediation, 10(2): 79-90.
GUPTA, V. K., A. K. SHRIVASTAVA \& N. JAIN. 2001. Biosorption of Chromium (VI) from Aqueous Solutions by Green Algae Spirogyra Species. Water Research, 35(17): 4079-4085.

HAN, F. X., B. B. MARUTHI-SRIDHAR, D. L. MONTS \& Y. SU. 2004. Phytoavailability and Toxicity of Trivalent and Hexavalent Chromium to Brassica juncea. New Phytologist, 162(2): 489-499.

HOAGLAND, D. R. \& H. I. ARNON. 1950. The Water Culture Method for Growing Plants Without Soil. California Agricultural Experimental Station Circular.

KSHEMINSKA, H., D. FEDOROVYCH, L,. BABYAK, D,. YANOVYCH, P. KASZYCKI \& H. KOLOCZEK. 2005. Chromium (III) and (VI) Tolerance and Bioaccumulation in Yeast: A Survey of Cellular Chromium Content in Selected Strains of Representative Genera. Process Biochemistry, 40: 1565-1572.

KUMAR-PANDA, S. 2007. Chromium-Mediated Oxidative Stress and Ultrastructural Changes in Root Cells of Developing Rice Seedlings. Journal of Plant Physiology, 164: 1419-1428.

LAGE-PINTO, F., J. G. OLIVEIRA, DA CUNHA, M., SOUZA, C. M. M., C. E. REZENDE, R. A. AZEVEDO \& A. P. VITÓRIA. 2008. Chlorophyll a Fluorescence and Ultrastructural Changes in Chloroplast of Water Hyacinth as Indicators of Environmental Stress. Environmental and Experimental Botany, 64: 307-313.

LARCHER, W. 1995. Ecological Plant Physiology. Springer Verlag. Berlín.

MAINE, M. A., N. L. SUÑE \& S. C. LAGGER. 2004. Chromium Bioaccumulation: Comparison of the Capacity of Two Floating Aquatic Macrophytes. Water Research, 38: 1494-1501.

MALIK, A. 2007. Environmental Challenge Vis a Vis Opportunity: The Case of Water Hyacinth. Environment International, 33: 122-138.

MANDIWANA, K. L., N. PANICHEV, M. KATAEVA \& S. SIEBERT. 2007. The Solubility of Cr (III) and $\mathrm{Cr}$ (VI) Compounds in Soil and Their availability to Plants. Journal of Hazardous Materials, 147: 540-545.

MANGABEIRA, P. A. O., L. LABEJOF, A. LAMPERT, A. A. F. DE ALMEIDA, A. H. OLIVEIRA, F. ESCAIG, M. I. G. SEVERO, D. DA SILVA, M. SALOES, M. MIELKE, E. R. LUCENA, M. C. MARTINS, K. B. SANTANA, K. L. GAVRILOV, P. GALLE \& R. LEVI-SETTI. 2004. 
Accumulation of Chromium in Root Tissues of Eichhornia crassipes (Mart.) Solms. in Cachoeira River-Brazil. Applied Surface Science, 231-232: 497-501.

OSPINA-ALVAREZ, N., PEÑA-SALAMANCA, E. \& BENITEZ, R. 2006. Efecto de la salinidad en la capacidad de bioacumulación de plomo en el alga verde Rhizoclonium riparium (Roth) Harvey (Chlorophyceae, Cladophorales). Acta Biologica, 28: 17-25.

PARIS, C., H. HADAD, M. A. MAINE \& N. SUÑE. 2005. Eficiencia de dos macrófitas flotantes libres en la absorción de metales pesados. Limnetica, 24(3-4): 237-2-44.

PATEL, S. 2012. Threats, Management and Envisaged Utilizations of Aquatic Weed Eichhornia crassipes: An Overview. Review of Environmental Science and Biotechnology, 11: 249-259.

PEÑA-SALAMANCA, E., A. L. RENGIFO-GALLEGO \& N., BENITEZ-CAMPO. 2011. Detoxification Mechanisms of Heavy Metals by AlgalBacteria Consortia. En: Handbook of Marine Macroalgae: Biotechnology and Applied Phycology. K. Se-Kwon (ed.): 441-452. JohnWiley \& Sons, Chichester.

PEÑA-SALAMANCA, E., J.R. CANTERA \& E. MUÑOZ. 2012. Evaluación de la contaminación en ecosistemas acuáticos: un estudio de caso en la laguna de Sonso, cuenca alta del Río Cauca. Programa Editorial Universidad del Valle.

PETTINE, M., L. D'OTTONE, L. CAMPANELLA, F. J. MILLERO \& R. PASSINO. 1998. The Reduction of Chromium (VI) by Iron (II) in Aqueous Solutions. Geochimica et Cosmochimica Acta, 62(9): 1509-1519.

PETTINE, M., D. TONNINA \& F. J. MILLERO. 2006. Chromium (VI) Reduction by Sulphur (IV) in Aqueous Solutions. Marine Chemistry, 99: $31-41$.

SANITA DI TOPPI, L., E. VURRO, L. ROSSI, R. MARABOTTINI, R. MUSETTI, M. CARERI, M. MAFFINI, C. MUCCHINO, C. CORRADINI \& M. BADIANI. 2007. Different Compensatory Mechanisms in Two Metal-Accumulating Aquatic Macrophytes Exposed to Acute Cadmium Stress in Outdoor Artificial Lakes. Chemosphere, 68: 769-80.
SANKAR-GANESH, K., L. BASKARAN, K. RAJASEKARAN, K. SUMATHI, A. L. A. CHIDAMBARAM \& P. SUNDARAMOORTHY. 2008. Chromium Stress Induced Alterations in Biochemical and Enzyme Metabolism in Aquatic and Terrestrial Plants. Colloids and Surfaces B: Biointerfaces, 63: 159-163.

VARDANYAN, L. \& B. INGOLE. 2006. Studies on Heavy Metal Accumulation in Aquatic Macrophytes from Sevan (Armenia) and Carambolim (India) Lake Systems. Environment International, 32: 208-218.

VARÓN-LÓPEZ, A. 2009. Dinámica y Distribución de la Contaminación por Cromo, Plomo y Mercurio, en Especies Representativas de la Laguna de Sonso. (Cali-Colombia). Tesis de grado en Química. Universidad del Valle, Colombia.

VITÓRIA, A. P., F. LAGE-PINTO, L. B. CAMPANELI DA SILVA, M. DA CUNHA, J. GONCALVES DE OLIVEIRA, C. E. REZENDE, C. M. MAGALHAES DE SOUZA \& R. A. AZEVEDO. 2011. Structural and Ecophysiological Alterations of the Water Hyacinth [Eichhornia crassipes (Mart.) Solms] Due to Anthropogenic Stress in Brazilian Rivers. Brazilian Archives of Biology and Technology: An International Journal, 54(5): 1059-1068.

WANG, H. \& JIA Y. 2009. Bioaccumulation of Heavy Metals by Phragmites australis Cultivated in Synthesized Substrates. Journal of Environmental Sciences, 21(10): 1409-1414.

WANG, W. \& M. A. LEWIS. 1997. Metal Accumulation by Aquatic Macrophytes. En: Plants for Environmental studies. W. Wang, J. W. Gorsuch \& J. S. Hughes (eds.): 367-416. CRC Press. Lewis Publishers. Nueva York.

WEERASINGHE, A., S. ARIYAWNASA \& R. WEERASOORIYA. 2008. Phytoremediation Potential of Ipomoea aquatica for Cr (VI) Mitigation. Chemosphere, 70: 521-524.

XIE, Y. \& D. YU. 2003. The Significance of Lateral Roots in Phosphorus (P) Acquisition of Water Hyacinth (Eichhornia crassipes). Aquatic Botany, 75: 311-321.

ZAYED, A. M. \& N. TERRY. 2003. Chromium in the Environment: Factors Affecting Biological Remediation. Plant and Soil, 249: 139-156. 